\title{
Mobile Based Study Links Insomnia and Sympathovagal Balance
}

\author{
Shuli Eyal, Anda Baharav \\ HypnoCore LTD, Petach-Tikva, Israel
}

\begin{abstract}
Insomnia is a high prevalence sleep disorder. Current theories point towards the role of cognitive and physiological hyperarousal in the pathophysiology of Insomnia. Our aim was to investigate the potential application of autonomic nervous system viewed through the prism of heart rate variability for understanding and assessing insomnia.

Inter beat intervals were recorded using a mobile app, SleepRate, and an off-the-shelf sport belt. 98 users who participated in a sleep assessment and therapy program were labeled by the app as insomniacs (IN). They were compared to 250 users who just monitored their sleep. Heart-rate variability analysis was performed for each night. Mean heart-rate in IN was significantly higher than in reference group (RG). Sympathovagal balance values for each of the different sleep stages and for the period prior to sleep onset were significantly higher in IN compared to the RG.

Mobile technology enabled very large scale, measurement of physiological signals. The findings indicate an increased sympathetic predominance in IN. This is consistent with recent theories linking physiological hyperarousal and insomnia. The autonomic approach to sleep evaluation may be a useful alternative to the gold standard whole-night PSG for poor sleepers.
\end{abstract}

\section{Introduction}

Epidemiological studies indicate a $10 \%$ prevalence of chronic insomnia as a syndrome [1]; and $30-48 \%$ of adults are complaining of some insomnia symptoms [2]. While the pathophysiology of this widespread ailment is multifactorial and not completely understood, current theories and research results [3,4] suggest cognitive and physiological hyperarousal play a significant role in insomnia.

Most previous studies were performed in sleep laboratories, a very un-natural sleep environment, and others rely on subjective information only. We aimed at investigating the role of the autonomic nervous system in insomnia using the noninvasive heart rate variability (HRV) approach, and mobile technology that allows obtaining large scale data in the habitual sleep environment.

\section{Method}

Data have been obtained from users of a sleep monitoring, evaluation and therapy service offered via a mobile application (SleepRate). De-identified measured sleep data acquired using a chest strap heart rate monitor (HRM) (H7-Polar), and reported data have been analyzed.

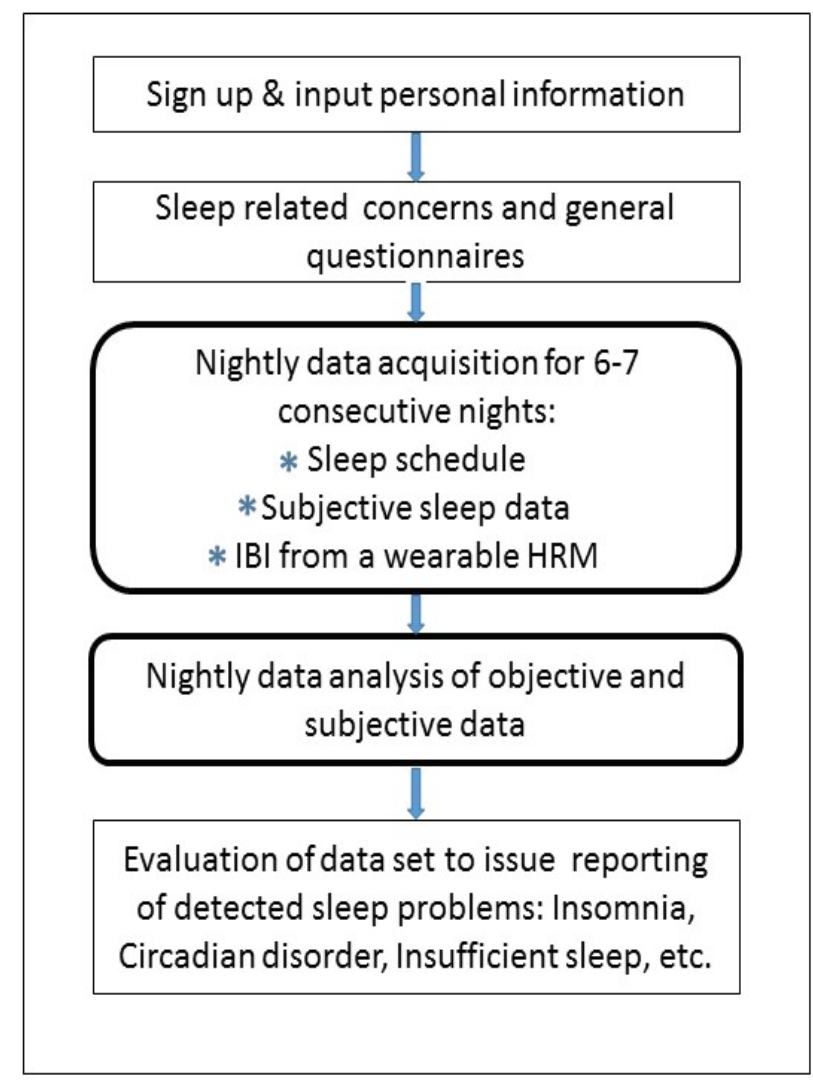

Figure 1. App flowchart 
App users have the choice of either simply monitoring their nights, or engaging in a sleep assessment and selfhelp therapy program. The assessment procedure is built up of 3 steps (see Figure 1): (i) first at sign up includes personal info and general sleep questionnaires and concerns; (ii) the second step requires actual sleep information, both objective and subjective, for a week; (iii) last step is an automatic evaluation of the initial information and night by night sleep data acquired during the assessment, to yield a report regarding detected sleep problems that need attention, namely Insomnia, Circadian Rhythm Disorders, Insufficient Sleep, or a suspected underlying clinical sleep problem. The second step is essential, as during at least 6 out of 7 consecutive nights the user monitors his/hers sleep. Data include bed time and wake up time, perceived sleep/wake durations, sleep satisfaction, daytime stress, and sleepiness levels. Objective sleep variables are obtained from inter-beatinterval (IBI) analysis acquired with a BLE chest HRM. This analysis has been validated [5], and results in objective values of sleep duration, sleep fragmentation, falling asleep time and sleep stages, as well as night time stress [6].

Our main goal in this study was to characterize the objective sleep variables of people with Insomnia who sleep in their natural sleep environment. Thus we chose to look into the following groups:

A. Insomnia Group (IN) - 98 users engaged in the program and found to suffer of Insomnia. This group contributed a total of 581 assessment nights for analysis.

B. Reference Group (RG) - 250 users who monitored their sleep for at least 6 nights, a total of 1500 monitoring nights with only their first 6 tracked nights included in this study.

For each night we looked into the following parameters: Subjective sleep parameters: (1) Subjective sleep onset (SO-S) - which is the user's estimation of how long was his falling asleep time, (2) Subjective wake after sleep onset (WASO-S) - the user's estimation of how long was he awake after falling asleep, (3) Subjective sleep efficiency (SE-S) - based on the previous two parameters we calculate the percentage of time the user estimated that he was asleep out of the time he intended to sleep.

Objective sleep parameters from the HRV sleep stages analysis. This included: (1) Objective sleep onset (SO-O), (2) Objective wake after sleep onset (WASO-O), and (3) Objective sleep efficiency (SE-O). (4) Autonomic arousals index (AI), which is the number of series of increasing $\mathrm{HR}$ per hour of sleep; the duration used here correlates well with cortical arousals.

Heart rate (HR) parameters in the time domain that were compared include: (1) Mean heart rate (HRASO) calculated for the time after sleep onset and before wake up, and (2) Resting heart rate (REST-HR), defined as the minimal mean HR in a window of 10 minutes during the night.

HR parameters in the time-frequency domain: The sympathovagal balance (SVB), which is represented by the Low Frequency to the High Frequency range power content ratio of the HRV spectrum [7] averaged for each of the different sleep stages after falling asleep and for the period prior to falling asleep.

Significant difference was considered for the two-tailed unpaired t-test with $\mathrm{p}>0.05$.

\section{Results}

IN group: 60\% male, mean age 46.6 (SD 13.0), mean BMI 24.9 (SD 4.7). No demographic information was collected for RG.

\subsection{Sleep parameters:}

Average subjective and objective values for each of the groups are presented in Table 1 . There was no significant difference between the SO-S of the two groups. WASO-S in IN users was significantly higher than in RG group. Note that the variability of WASO-S in IN users was also higher than in those of RG. SE-S was significantly lower in IN compared to RG, again with much higher variability in the IN group.

The first three objective parameters showed no significant differences between the groups. The AI was significantly higher for the RG as compared to the IN.

Table 1: Sleep parameters for both groups. Values are mean \pm SD. IN - Insomnia group and RG - Reference Group.

\begin{tabular}{llll}
\hline & $\begin{array}{c}\text { IN } \\
(\mathrm{N}=98)\end{array}$ & $\begin{array}{c}\text { RG } \\
(\mathrm{N}=250)\end{array}$ & p-value \\
\hline Subjective & & & \\
SO-S (min) & $22.1 \pm 16.3$ & $22.2 \pm 13.2$ & NS- \\
WASO-S (min) & $35.7 \pm 27.3$ & $18.5 \pm 12.9$ & $<0.0001$ \\
SE-S (\%) & $86.4 \pm 8.3$ & $90.6 \pm 4.3$ & $<0.0001$ \\
& & & \\
Objective & & & \\
SO-O (min) & $19.7 \pm 7.4$ & $21.0 \pm 10.1$ & NS \\
WASO-O (min) & $51.5 \pm 14.1$ & $49.1 \pm 11.1$ & NS \\
SE-O (\%) & $84.7 \pm 3.4$ & $84.8 \pm 3.1$ & NS \\
AI & $13.0 \pm 5.7$ & $14.2 \pm 3.1$ & $<0.05$ \\
\hline
\end{tabular}

\subsection{Heart rate parameters}

Both HRASO and REST-HR in IN users were significantly higher than in RG users. 
In addition both parameters showed higher variability between the users in IN compared to RG users.

Table 2: Time domain parameters for both groups. Values are mean \pm SD. IN - Insomnia group and RG - Reference Group.

\begin{tabular}{lccc}
\hline & IN & RG & p-value \\
& $(\mathrm{N}=98)$ & $(\mathrm{N}=250)$ & \\
\hline HRASO (bpm) & $62.4 \pm 9.1$ & $59.5 \pm 5.0$ & 0.0002 \\
REST-HR (bpm) & $56.3 \pm 8.5$ & $53.6 \pm 4.5$ & 0.0002 \\
\hline
\end{tabular}

\subsection{HR parameters - time-frequency domain}

SVB values were significantly higher in IN compared to RG for each of the different HRV defined sleep stages after sleep onset and for the period prior to sleep onset (Figure 2).

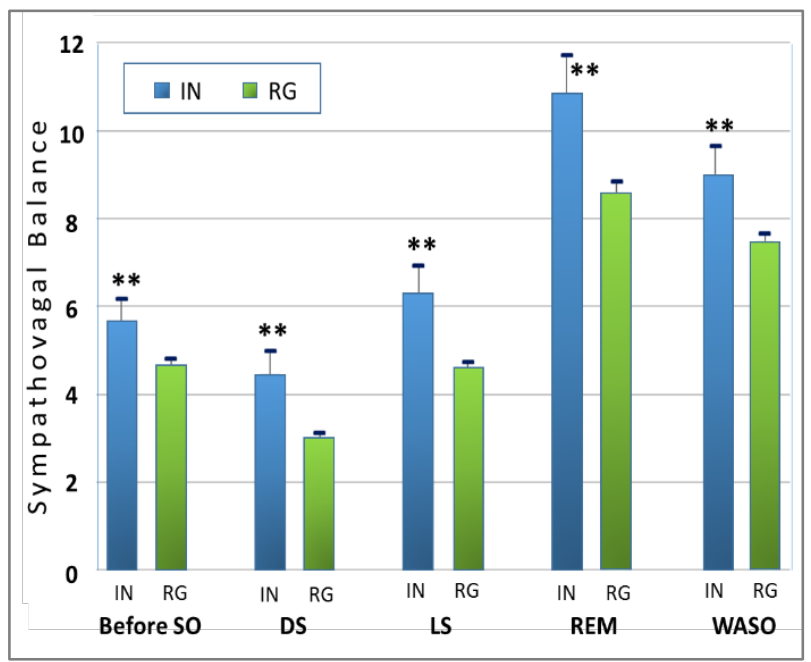

Figure 2: Sympathovagal Balance (Mean+SE) for the period before sleep onset (Before SO) and every sleep stage (DS - deep sleep, LS - light sleep, REM sleep, WASO - wake after sleep onset) for the 2 groups. In each pair of bars the left is Insomnia group (IN) and the right is Reference Groups (RG).

** Significant difference $(\mathrm{p}<0.01)$

\section{Conclusion}

Subjects in the IN group presented with a significantly lower subjective sleep efficiency than the efficiency of the RG. While there was a very similar average sleep latency (SO) in the two groups, time spent awake (WASO) was on average twice larger than the same variable in the RG. The variability between users was higher in all variables for the IN subjects, suggesting that those subjects oscillated between bad and good nights, and thus their average per user can range from normal values to extremely abnormal ones.

No significant difference between groups has been detected in the objective sleep variables, indicating that sleep-wake objective macrostructure variables by themselves are not sufficient to uncover Insomnia.

Interestingly enough, both the mean night $\mathrm{HR}$ and the basal HR (REST-HR) were significantly higher (by almost $3 \mathrm{bpm}$ ) in the IN group. This finding indicates that Insomniacs have a lower fitness level, and that their heart works a bit harder than the heart of those in the RG, at least at night.

The Insomniacs also displayed a higher SVB during all sleep stages, indicating a high stress level in this group. Moreover, the SVB was higher in Insomniacs before falling asleep, indicating a higher "alertness" in this group. These findings support the hyperarousal theory of Insomnia, sufferers have higher HR and also increased stress. This may explain, at least partly, their different estimation of sleep and wakefulness, their feeling that they are awake longer at night than their normal sleepers counterparts.

AI represents another aspect of good night sleep, and it is independent of the macro sleep architecture, which relates to the relative durations of different sleep stages during the night. The increased AI in RG suggest that sleep fragmentation does not explain the subjective poor sleep of IN users. Also AI is not a contribute to the higher SVB in the IN group

The above findings are in agreement with Bonnet and Arand [8]. Their study was performed over a carefully selected limited number of users who either presented relatively sever symptoms of Insomnia, or were verified as being normal sleepers. Using the advantages of the mobile app, namely accessibility to large-scale measurements, and the ability to perform several tests for the same user in his/her habitual sleep environment, the current study was able to show that the same results hold for the IN group in general and in a more realistic environment. The main limitation of this study is the lack of demographics for the RG, which, although unlikely, may have a contribution to the differences between the groups.

These clear differences between the insomniac group and their reference group suggest that the autonomic approach to sleep evaluation can be a useful alternative to the gold standard whole night PSG for poor sleepers.

\section{References}

[1] Ohayon M. Epidemiology of insomnia: what we know and what we still need to learn. Sleep Med Rev 2002;6:97-111.

[2] Morin CM, LeBlanc M, Daley M, Gregoire JP, Merette C. Epidemiology of insomnia: Prevalence, self-help 
treatments, consultations, and determinants of help-seeking behaviors. Sleep Medicine 2006;7:123-130.

[3] Bastien CH, St-Jean G, Morin CM, Turcotte I, Carrier J. Chronic psychophysiological insomnia: hyperarousal and/or inhibition deficits? An ERPs investigation. Sleep 2008;31(6):887-898.

[4] Bonnet MH, Arand DL. Hyperarousal and insomnia: State of the science. Sleep Med. Rev. 2010:14:9-15

[5] Decker MJ, Eyal S, Shinar Z, Fuxman Y, et al. Sleep architecture and ventilation derived through analyses of the electrocardiogram. Sleep Breath. 2010;14:233-239.

[6] Eyal S, Dagan Y, Baharav A. Sleep in the cloud: On how to use available heart rate monitors to track sleep and improve quality of life. Computing in Cardiology 2012; 39:329-332.
[7] Pagani M, Furlan R, Pizzinelli P, Crivellaro W, Cerutti S, Malliani A. Spectral analysis of R-R and arterial pressure variabilities for the assessment of sympathovagal interaction during mental stress in humans. J Hypertens 1989;7(suppl 6):S14-S15.

[8] Bonnet MH, Arand DL. Heart rate variability in insomniacs and matched normal sleepers. Psychosomatic Med. 1998:60(5):610-5.

Address for correspondence.

Shuli Eyal.

20 Hamagshimim St,

Petach Tikva, 4934829, Israel.

shuli@sleeprate.com 